\title{
Analysis of Mechanical Behavior of Red Cell Membrane in Sickle Cell Disease
}

\author{
Demeke Fisseha*, V. K. Katiyar \\ Department of Mathematics, Indian Institute of Technology Roorkee, IIT Roorkee, 247667, India
}

\begin{abstract}
Sickle cell disease (SCD) is a disease of abnormal rheology. The rheological properties of normal erythrocytes appear to be largely determined by those of the red cell membrane. In SCD, the intracellular polymerization of sickle hemoglobin upon deoxygnation leads to marked increase in intracellular viscosity and elastic stiffness and also having indirect effects on cell membrane .To examine mathematically, the abnormal cell rheology behavior due to polymerization process and that due membrane abnormalities, we mechanically modeled the whole cell deformability as viscoelastic solid and proposed a Voigt-model of nonlinear viscoelastic solid constitutive relation as " mixture" of an elastic and viscous dissipative parts, with parameters of elastic and viscous moduli. The elastic part used to express stress-strain relations via strain energy function of the material and the viscous part derivation depends on strain - rate of deformation. The combination of both constitutive expressions is used to predict the viscoelastic properties of normal and sickle erythrocyte. Furthermore, sickle hemoglobin polymerization also leads to alter the osmotic behavior of the cell and to investigate such osmotic effect; we employ the van't Hoff law of osmotic pressure versus volume relation. The analysis of both formulations presented well the abnormal rheological/mechanical characterization of sickle erythrocyte membrane as we understood and concluded from our results.
\end{abstract}

Keywords Sickle cell disease, RBC deformability, Nonlinear viscoelasticity, Constitutive relation, Osmotic behaviour

\section{Introduction}

The deformability of erythrocytes and its membrane is critical determinant of blood flow in the microcirculation. This deformability is the combined result of several mechanical and geometrical properties, such as internal viscosity, membrane elasticity and viscosity, and surface area volume ratio[1]. In each red blood cell two $\beta$ - globin proteins combine with two $\alpha$-globin proteins and a central heme molecule to form adult hemoglobin ( $\mathrm{HbA})$. A healthy human red blood cell (RBC) contains approximately 250 million hemoglobin $(\mathrm{Hb})$ molecules[2], totaling $25-30 \%$ of the cell [3]. Sickle cell disease (SCD), a molecular disease[4] is caused by a single point mutation that substitutes valine for glutamic acid at the sixth position in the $\beta$-globin chain, resulting sickle hemoglobin (HbS). Therefore, in sickle cell hemoglobin $(\mathrm{HbS})$, the normal sequence of Val-His-LeuThr-Pro-Glu-Glu-Lys is changed to Val-His- Leu-Thr- ProVal-Glu-Lys, with the amino acid valine substituting for the glutamic acid in the $\beta 6$ site[2,5]. The replacement of two charged groups by two hydrophobic ones leads to polymerizationof deoxygenated $\mathrm{Hb}$ and to formation of long stiff

* Corresponding author:

demekef12@yahoo.com (Demeke Fisseha)

Published online at http://journal.sapub.org/am

Copyright (C) 2012 Scientific \& Academic Publishing. All Rights Reserved rod like fibers[3,6] which force red blood cells (RBCs) to form a sickle shape .The sickle- shaped RBC are not able to deform sufficiently to pass through small blood vessels.The mutation causes a defect in the hemoglobin structure which is responsible for profound changes in molecular stability and solubility. In sickle cell disease (SCD), the red blood cells (RBCs) exhibit reduced deformability, and this abnormality in the erythrocyte rheology are of clinical importance[1].

In sickle cell syndromes, abnormal rheological properties are caused primarily by the increase in internal red cell viscosity due to the aggregation or polymerization of sickle hemoglobin (HbS) upon deoxygenation, as well as biochemical abnormalities in the membrane of the sickle erythrocytes[7]. These membrane changes, both reversible and irreversible, are thought to occur due to repeated cycles of polymerization - depolymerization during the life of the sickle erythrocyte circulation[8]. The life span of sickle cells are very much reduced to about one-tenth that of normal cells, some 10-20days[4]. Other rheological abnormalities may occur because of the shrinkage of certain sickle cells (the dense cells) so that they have a very high mean corpuscular hemoglobin concentration (MCHC) $(>40 \mathrm{~g} / \mathrm{dl})$ which elevates internal viscosity, even of the oxygenated $\mathrm{HbS}$. Lew and Bookchin[9] perform a theoretical analysis of the possible osmotic effects of deoxy-HbS polymerization. Polymerization of deoxygenation HbS reduces the cell concentra- 
tion of osmotically active particles, and also assumed to induce cell shrinkage[10]. Dong et al. [7] developed a simple mathematical model of whole cell deformability in narrow vessel wall to estimate the components of abnormal cell rheology due to the polymerization process and that due to the membrane abnormalities. The model uses hydrodynamic lubrication theory to describe the pulsatile flow in the gap between a cell and the vessel wall. The result shows the transition from membrane to internal polymer dominance of deformability as oxygen saturation is lowered. Chien et al.[11] have investigated that the viscoelastic behavior of sickle cell anemia RBC suspensions at low oxygen saturation is dominated by the RBC cytoplasmic properties. Drasler et al.[12] suggest that the presence of both the cell membrane and the internal $\mathrm{HbS}$ is a major factor in determining the viscoelastic behavior of sickle cells during deoxygenation. Pathogenesis in SCD arises from the deoxygenation dependant polymerization of sickle cell hemoglobin $(\mathrm{HbS})$ into long, stiff, rod-like fibres[13]. Maciaszek and Lykotrafitis[5] employed AFM to measure the stiffness of abnormal human RBCs from human subjects with the genotype for skill cell trait, and they conclude that the young's modulus of pathological erythrocytes approximately three times higher than in normal cells. The differences indicate that the effect of the polymerization of sickle hemoglobin as well as possible changes in the organization of the cell cytoskeleton associated with the sickle cell trait. Evan and Hochmuth[14] develop a theory for viscoelastic behavior of large membrane deformations and apply the analysis to the relaxation of projections produced by small micropipette aspiration of red cell discocytes. The membrane stiffness, the cell geometry and the viscoelastic components are influencing factors on survival and functionality of erythrocytes. As Martorana et al. [15] review that only few studies have analysed the viscoelastic characteristics of red blood cells, even less are the studies on patients affected by sickle cell disease (SCD), a pathology characterized by acute and chronic impairment of cell flexibility due to the formation of intracellular sickle hemoglobin $(\mathrm{HbS})$ polymers. Moreover, Martorana et al. have investigated the behavior of the blood in SCD, from an original rheological point of view, by evaluating the viscoelastic properties of sickle cell in oscillating harmonic sinusoidal model. And also Messman et al. [16] evaluate the mechanical properties of sickle erythrocyte membrane in ektacytometer. However, as our consideration regarding to the literature review, there may not be any mathematical analysis to this study especially related to membrane theory and viscoelastic behavior of the material to quantify the mechanical properties of sickle erythrocyte membrane.

In the present study, by proposing a suitable new mathematical formulation, we try to analyse and show the mechanical characterization of sickle cell membrane associated with sickle cell hemoglobin by considering change of numerical values of membrane shear modulus and viscosity, and strain - rate. The change of osmotic pressure versus volume relation of the cell also considered for the same purpose.

\section{Mathematical Formulation}

A red blood cell (RBC) is mainly composed of an inner liquid (hemoglobin solution) and a membrane (boundary of the cell). Comparing with membrane, the resistance of the inner liquid to external stress is small and negligible[17]. Therefore, the rheological properties of normal RBCs appear to be largely determined by the red cell membrane .Altered membrane properties in SCD play a primary role in the altered rheology that characterizes the disease and that tied directly to sickle hemoglobin polymerization[18]. Mean that, in sickle cell, assumed that the viscous behavior is dominated by the cell interior, however, having indirect effect on the cell membrane[7]. In sickle cell disease (SCD) the intracellular polymerization of sickle cell hemoglobin upon deoxygenation leads to marked increase in internal viscosity, and elastic stiffness. The interior of the cell is modeled as a voigt viscoelastic solid with parameters for the viscous and elastic modulus while the membrane is assigned an elastic shear modulus[7]. As Pucci and Saccomadi[19] reviewed that the Kelvin-Viogt models of non-linear viscoelastic constitutive equations for the Cauchy stress may be split in the sum of two terms: an elastic part and dissipative part .An incompressible viscoelastic continuum is adopted for the cell interior[7]. Thus, the generalization of an incompressible Voigt viscoelastic solid model can be thought of as a "mixture" in the following sense,

$$
T_{i j}=-P \delta_{i j}+T_{i j}^{e}+T_{i j}^{v}
$$

where $T_{i j}^{e}$ is the stress associated with an elastic component that can be expressed as a function of strain, and $T_{i j}^{v}$ the stress associated with viscous fluid depend on rate of deformation, $-P \delta_{i j}$ is the reaction stress due to the incompressibility.

A general viscoelastic constitutive equation for erythrocyte membrane may be written in terms of principal strains and stresses. This implies no loss of generality, assuming that the membrane is isotropic in its own plane. Let deformation of the membrane is described by initial lengths $\mathrm{dx}_{1}$, $\mathrm{dx}_{2}$, and current lengths $\mathrm{dy}_{1}, \mathrm{dy}_{2}$ in a Cartesian coordinate system parallel (tangent) to the local principal direction. The extension ratios $\lambda_{1}$ and $\lambda_{2}$ are defined as ratios of final to initial lengths

$$
\lambda_{1}=\frac{d y_{1}}{d x_{1}} \quad \text { and } \quad \lambda_{2}=\frac{d y_{2}}{d x_{2}}
$$

The Green's strain tensor for large deformations is defined by

$$
e_{11}=\frac{1}{2}\left(\lambda_{1}^{2}-1\right) \quad, \quad e_{22}=\frac{1}{2}\left(\lambda_{2}^{2}-1\right)
$$

Because of choosing the principal axis coordinate system (where the deformation of the material is simply extension and compression along the axes), thus the strain tensors different from the axes are assumed to zero (i.e. $e_{12}=e_{21}=0$ )

From equation (1) the stress term, $T_{i j}^{e}$ the elastic part of the viscoelastic membrane material that can be derived from strain energy function, $W$ of the material, and relate the stress 
to strain via $W$.The stress within the membrane may be thought of as a generalization of the surface tension[20]. When the membrane is treated as $2 \mathrm{D}$ elastic continuum, $W$ $\left(\lambda_{1}, \lambda_{2}\right)$ denoted the strain energy per unit area. The principal membrane tensions $T_{i}$ are thus expressed by

$$
T_{i}=\frac{1}{\lambda_{1} \lambda_{2}} \lambda_{i} \frac{\partial W}{\partial \lambda_{i}} \quad(i=1,2)
$$

Deformability is generally described as the ability of a body to change its shape in response to a deforming force. The deformability of the RBC is a primary determinant of blood behavior. In the microcirculation, the ability of the $\mathrm{RBC}$ to deform is particularly crucial because RBCs must pass through narrow capillaries that have diameters half the size of their own. Normal RBCs are modeled as viscous liquid drops surrounded by elastic membranes, and their deformability depends on the viscosity of the cytoplasmic fluid and on the rigidity of the cell membrane[21].

In case of sickle cell disease (SCD), the intracellular polymerization of sickle hemoglobin upon deoxygenation leads to a marked increase in an elastic stiffness and intracellular viscosity [7].

Mechanically, sickle cell membranes differ from normal RBC membranes in their static and dynamic rigidities [18].Altered membrane properties in SCD play a primary role in altered rheology that characterizes the disease and tied directly to sickle hemoglobin polymerization[7]. The presence of polymerized sickle cell hemoglobin increases cytoplasmic viscosity and decreased deformability[18]. Loss of deformability in sickle RBCs significantly increases the viscosity of the whole blood, and is, arguably, the most significant pathobiomechanical alteration resulting in vaso-occlusion[18,22].

A critical point of SCD is represented by the rheological alterations of sickle cells determined by the transition from sol to gel hemoglobin producing a dramatic change in cell viscosity and viscoelastic properties [15].

We can obtain a better understanding of the viscoelastic behavior of red blood cell (RBC) membrane in normal[14] and in sickle cell disease[7,18] states by employing fundamental principles that govern viscoelastic solids. Based on this consideration, mechanically, in the present model we assume that the membrane behaves like a neo-Hookean solid material. Therefore, for a 2D neo-Hookean membrane, the strain energy function is given by[23]

$$
W=\frac{G h}{2}\left(I_{1}+2+\frac{1}{I_{2}+1}\right)
$$

Where $\mathrm{G}$ is the shear modulus of elasticity of the membrane, $\mathrm{h}$ is the thickness. The two-dimensional strain invariant $I_{1}$ and $I_{2}$ defined by Skalak et al. [24] as

$$
I_{1}=\lambda_{1}^{2}+\lambda_{2}^{2}-2 \text { and } I_{2}=\lambda_{1}^{2} \lambda_{2}^{2}-1
$$

Where $\lambda_{1}$ and $\lambda_{2}$ are the principal stretch ratios as defined in equation (2). The tensions $T_{1}$ and $T_{2}$ along the principal directions using equations (4), (5) and (6) are,

$$
T_{1}=\frac{G h}{\lambda_{1} \lambda_{2}}\left(\lambda_{1}^{2}-\frac{1}{\left(\lambda_{1} \lambda_{2}\right)^{2}}\right)
$$

$$
T_{2}=\frac{G h}{\lambda_{1} \lambda_{2}}\left(\lambda_{2}^{2}-\frac{1}{\left(\lambda_{1} \lambda_{2}\right)^{2}}\right)
$$

The viscous stress term $T_{i j}^{v}$ from equation (1) as Evan and Hochmuth [14] can be defined a constant times the rate of deformation tensor as follows

$$
T_{i j}^{v}=2 \eta_{m} V_{i j}
$$

Where $\eta_{m}$ which is the coefficient of viscosity, $V_{i j}$ is rate of deformation tensor. The rate of deformation tensor of $V_{i j}\left(\right.$ second $\left.^{-1}\right)$ is defined by the time rate of change of the squared lengths that occur as a body deforms from the reference to the current configuration in a material coordinate system. The difference between the squared lengths in the deformed and initial (undeformed) coordinate system is defined as

$$
\begin{aligned}
(d s)^{2}-\left(d s_{0}\right)^{2} & =d y_{k} d y_{k}-d x_{k} d x_{k} \\
& =2 e_{i j} d x_{i} d x_{j}
\end{aligned}
$$

By taking the time derivative both sides of equation (10) we obtain

$$
\frac{d}{d t}(d s)^{2}=2 \quad \dot{e}_{i j} d x_{i} d x_{j}
$$

we have the fact that $\left(d s_{0}\right), d x_{i}$ and $d x_{j}$ are constants, and $\dot{e}_{i j}$ indicates the partial derivative of Green- Lagrangian strain tensor with respect to time. And also from right side of equation (11) we obtain

$$
\begin{aligned}
\frac{d}{d t}\left((d s)^{2}\right)=\frac{d}{d t}\left(d y_{k} d y_{k}\right) & =\left(\frac{\partial v_{i}}{\partial y_{j}}+\frac{\partial v_{j}}{\partial y_{i}}\right) d y_{i} d y_{j} \\
& =2 V_{i j} d y_{i} d y_{j}
\end{aligned}
$$

Where $v_{i}$ is the $i$ th component of the in-plane velocity field, from the combination of equation (11) and equation (12) we obtain

$$
\begin{gathered}
V_{i j} d y_{i} d y_{j}=\dot{e}_{k l} d x_{k} d x_{l} \\
\Rightarrow V_{i j}=\dot{e}_{k l} \frac{\partial x_{k}}{\partial y_{i}} \frac{\partial x_{l}}{\partial y_{j}}
\end{gathered}
$$

Then from equations 2,3 and 13 , the components of the rate of deformation tensors are given by

$$
V_{11}=\frac{1}{\lambda_{1}} \frac{d \lambda_{1}}{d t} \quad, \quad V_{22}=\frac{1}{\lambda_{2}} \frac{d \lambda_{2}}{d t}
$$

Therefore, from equation (9) and equation (14) we have

$$
T_{11}^{v}=\frac{2 \eta_{m}}{\lambda_{1}} \frac{d \lambda_{1}}{d t} \quad, \quad T_{22}^{v}=\frac{2 \eta_{m}}{\lambda_{2}} \frac{d \lambda_{2}}{d t}
$$

According to the principal axis system orientation, assuming that, $T_{i j}^{e}$ from equation (1) is the same as $T_{i}$ in equation (4) (i.e. $T_{i j}^{e}=T_{i}$ ). By using equations (7), (8), and (15), in equation (1) the membrane tensions versus strain and rate of strain undergoing simple extension (in the principal axis system) can be written as 


$$
\begin{aligned}
& T_{11}=-p+\frac{G h}{\lambda_{1} \lambda_{2}}\left(\lambda_{1}^{2}-\frac{1}{\left(\lambda_{1} \lambda_{2}\right)^{2}}\right)+\frac{2 \eta_{m}}{\lambda_{1}} \frac{d \lambda_{1}}{d t} \\
& T_{22}=-p+\frac{G h}{\lambda_{1} \lambda_{2}}\left(\lambda_{2}^{2}-\frac{1}{\left(\lambda_{1} \lambda_{2}\right)^{2}}\right)+\frac{2 \eta_{m}}{\lambda_{2}} \frac{d \lambda_{2}}{d t}
\end{aligned}
$$

If we assume the element of deformation experiences uniaxial tension, $T_{22}$ would equal to zero, and the result for $T_{11}$ is simply as follows

$$
T_{11}=-\frac{G h}{\lambda_{1} \lambda_{2}}\left(\lambda_{2}^{2}-\frac{1}{\left(\lambda_{1} \lambda_{2}\right)^{2}}\right)-\frac{2 \eta_{m}}{\lambda_{2}} \frac{d \lambda_{2}}{d t}+\frac{G h}{\lambda_{1} \lambda_{2}}\left(\lambda_{1}^{2}-\frac{1}{\left(\lambda_{1} \lambda_{2}\right)^{2}}\right)-\frac{2 \eta_{m}}{\lambda_{1}} \frac{d \lambda_{1}}{d t}
$$

Where $T_{11}$ the in-plane principal tension and "11" indicates the in-plane direction along the membrane and the unit normal of the plane on which the tension force is acting. Thus, during experiences of the in-plane membrane deformation, the membrane area is constraint, that is $\lambda_{1} \lambda_{2}=1$, (where $\lambda_{3}=1$ ), in this condition the in-plane shear modulus $\mu=G h$ throughout the entire deformation[25,26]. Therefore, equation (18) becomes

$$
T_{11}=\mu\left(\lambda_{1}^{2}-\frac{1}{\lambda_{1}^{2}}\right)+\frac{4 \eta_{m}}{\lambda_{1}} \frac{d \lambda_{1}}{d t}
$$

Where $\mu$ is the membrane shear elastic modulus and, $\eta_{m}$ is the membrane viscosity. These two parameters are important in biorheology of red blood cells (RBCs). The membrane elastic modulus $\mu$ represents the assessment of the elastic storage of energy primarily due to kinetic deformability of the erythrocytes while the viscous (loss)modulus $\left(\eta_{m}\right)$ is the assessment of rate of energy dissipation due to cell deformation[7]. In the normal RBC rheological property, the values of the parameters, that is, the membrane viscosity $\left(\eta_{m}=0.001 \mathrm{dyn} \mathrm{s} / \mathrm{cm}\right)$, membrane elastic modulus ( $\mu=0.01 \mathrm{dyn} / \mathrm{cm})$, and an experimental estimated strain rate $D \lambda_{1}=0.3 \mathrm{~s}^{-1}$.

In patient with $\mathrm{SCD}$, the presence of polymerized sickle hemoglobin, the RBC membrane viscosity and deformability different from those of normal erythrocytes[7,18]. During sickle hemoglobin gelation, the parameters are considered with the amount of polymer formed inside sickle cells or low oxygen saturation at a given $\mathrm{HbS}$ concentration[7]. As oxygen saturation is reduced, formation of $\mathrm{HbS}$ polymer could cause the development of a new cellular elastic- resistive element and a very large increase in cytoplasmic viscosity and in general $\mathrm{HbS}$ polymer shows viscoelastic solid nature[27]. On this abnormality condition, values of two intrinsic membrane viscoelastic properties (i.e. shear elastic modulus $\mu$, membrane surface viscosity $\eta_{m}$ ), both are similarly increased above normal[28]. Regarding to these change, sickled cell erythrocytes poorly deformable and this decrease in deformability leads to an increase in whole blood viscosity[15], and this clearly shows the decreasing of rate of strain. The membrane mechanical properties of irreversibly sickled cell (ISC) is more rigid ( $130 \%$ increased in $\mu)$ while the ISC membrane viscosity $\left(\eta_{m}\right)$ also increased with highly significant which is approximately $150 \%$ from those of the control RBC membrane[28]. Using this information we can estimate and prepare the following data table for our simulation purpose

*Note: $D \lambda_{1}=\frac{d \lambda_{1}}{d t}$ throughout this work.

Table1. Given and estimated values of the two parameters and rate of strain

\begin{tabular}{|c|c|c|c|}
\hline parameters & Given value & Reference & $\begin{array}{c}\text { Est.Values based on } \\
\text { polymer formation } \\
\text { increases }\end{array}$ \\
\hline $\begin{array}{c}\text { Shear elastic } \\
\text { modulus } \mu\end{array}$ & $0.01 \mathrm{dyn} / \mathrm{cm}$ & {$[14]$} & $0.012,0.023 \mathrm{dyn} / \mathrm{cm}$ \\
\hline $\begin{array}{c}\text { Membrane } \\
\text { viscosity } \eta_{m}\end{array}$ & $\begin{array}{c}0.001 \mathrm{dyn} \\
\mathrm{s} / \mathrm{cm}\end{array}$ & {$[14]$} & $\begin{array}{c}0.0015,0.0025 \mathrm{dyn} \\
\mathrm{s} / \mathrm{cm}\end{array}$ \\
\hline $\begin{array}{c}\text { Rate of strain } \\
\left(D \lambda_{1}\right)\end{array}$ & $0.3 \mathrm{~s}^{-1}$ & {$[26]$} & $0.2,0.01 \mathrm{~s}^{-1}$ \\
\hline
\end{tabular}

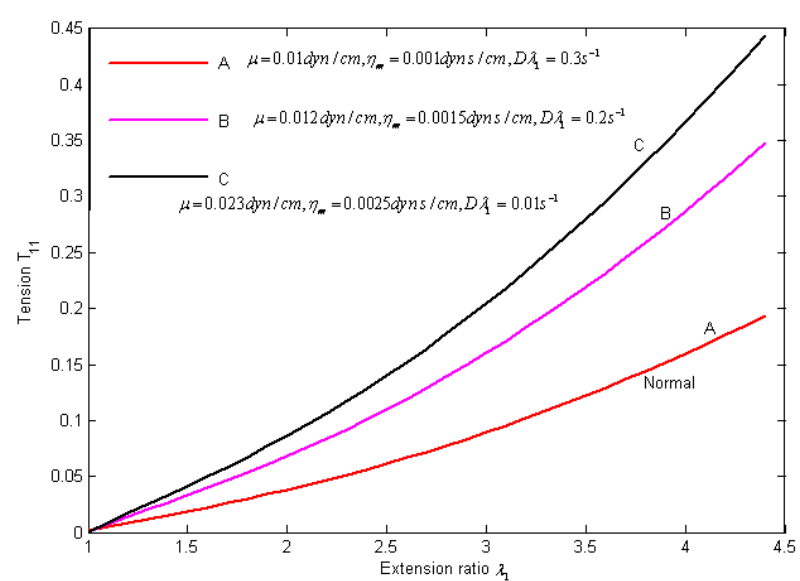

Figure 1. Stress versus strain and rate of strain curves in uniaxial tension. The different curve shows for various values of the parameters and strain rate from the table using equation (19)

\section{Osmotic Pressure - Volume Elations}

Sickle cell anemia is associated with a mutant hemoglobin, $\mathrm{HbS}$, which forms polymers in the RBCs of patients. RBCs adjust their volume according to the osmolality of the medium in which they are suspended. In Sickle cell anemia, red cells volume changes following hemoglobin $(\mathrm{S})(\mathrm{HbS})$ polymerization[7]. Polymerization of proteins with in cells and subcellular organelles may have powerful osmotic effects on hemoglobin molecule $(\mathrm{Hb})$ and may play a role in cell volume loss[7,29].Mathematically; the relationship between volume and osmotic pressure is expressed by van't Hoff law and is conventionally used to describe the osmotic behavior of living cells[30,31]

$$
P(V-b)=P_{0}\left(V_{0}-b\right)
$$

Where $P$ is the osmotic pressure, $V$ is the total cell volume, $b$ is osmotically inactive (non-solvent) volume, $V_{0}$ is the isotonic volume, and $P_{0}$ the isotonic osmotic pressure of the cell.

Because, the deoxygenated sickle cell has a decreased cell volume, decreased cell water and increased hemoglobin concentration, it resembles the "irreversibly" sickled cell. Thus, the cell shrinkage during sickling may play a role in the formation of "irreversibly" sickled cells[29]. 
Hemolysis is greatest in individual cells with the highest numbers of irreversibly sickled and dense cells [32]. Therefore, hemolysis associated with irreversible structural change of sickle cell erythrocytes. Thus, one can calculate a hemolytic volume $V_{H}$ and subsequently $P_{H}$ the osmotic pressure when the cell hemolysed, regarding to equation (20) we can write

$$
P_{H}=\frac{P_{0}\left(V_{0}-b\right)}{V_{H}-b}
$$

The kinetic osmotic pressure in sickle hemoglobin is lost upon deoxygenation. A loss of intracellular osmotic pressure during deoxygenation could dehydrate the erythrocyte sufficiently to promote more rapid sickle cell hemoglobin aggregation and polymerization[33].

In cells with the initial mean cell hemoglobin concentration (MCHC) of approximately $36 \mathrm{~g} / \mathrm{dl}, 60 \%$ polymerization reduces the osmotic contribution of $\mathrm{Hb}$, with $\approx 4 \%$ reduction in cell volume, where as with $\mathrm{MCHC}$ of $45 \mathrm{~g} / \mathrm{dl}$ (i.e. ISC level as Nash et al,[28] ) the osmotic contribution reduced in soluble $\mathrm{Hb}$, with $8 \%$ decrease in cell volume[9]. Of this, we understand again that, the osmotic behavior of $\mathrm{HbS}$ may significantly change during sickling[33].

Of particular interest, the change in osmotic pressure, $\Delta P$, which develops after the cell shrink with reversible change in sickle cell structure, and it can be written as

$$
\Delta P=P_{0}\left(V_{0}-b\right)\left(\frac{1}{V_{r}-b}-\frac{1}{V_{H}-b}\right)
$$

This is the gradient of osmotic pressure which the cell by being compressed enough by allowing its membrane going to be damage or direct removal of osmotic particles. $V_{r}$ is volume of the cell when first became shrink a reversible change in membrane structure, with the accompany of osmotic pressure as

$$
P_{r}=\frac{P_{0}\left(V_{0}-b\right)}{V_{r}-b}
$$

In SCD, abnormal irreversible sickle cell membrane elasticity is probably due to an irreversible alteration in membrane structure, distinct from a reversible, hemoglobin concentration dependent/membrane interaction that may influence membrane viscosity[28]. The cell shrink, when MCHC approached $40 \mathrm{~g} / \mathrm{dl}$, suggests that reversible $\mathrm{Hb}$ concentration dependent/ membrane interaction (i.e. a reversible change in membrane structure)[28] with $\approx 6 \%$ decrease in cell volume which is the mean of $\approx 4 \%$ and over $8 \%$ reduction in cell volume, corresponding to $\mathrm{MCHC} \approx 36 \mathrm{~g} / \mathrm{dl}$ and $45 \mathrm{~g} / \mathrm{dl}$ respectively[9]. Thus, mathematically, we conclude that, $V_{\mathrm{r}}=$ $V_{0}-0.06 V_{0}=0.94 V_{0}$. In addition, the isotonic osmotic pressure $P_{0}=300$ milliosmolar (mosmolar), and $\mathrm{b}=0.42 V_{0}$ osmotically inactive volume of the cell [30]. By substituting all such value in equation (22), we obtain

$$
\Delta P=334.615\left(\frac{\frac{V_{H}}{V_{0}}-0.94}{\frac{V_{H}}{V_{0}}-0.42}\right)
$$

Where $\frac{V_{H}}{V_{0}}$ is the volumetric ratio, which is between on an interval of $(0.42,0.94)$. Because the cell shrink or volume reduction is only on osmotically active parcticles.

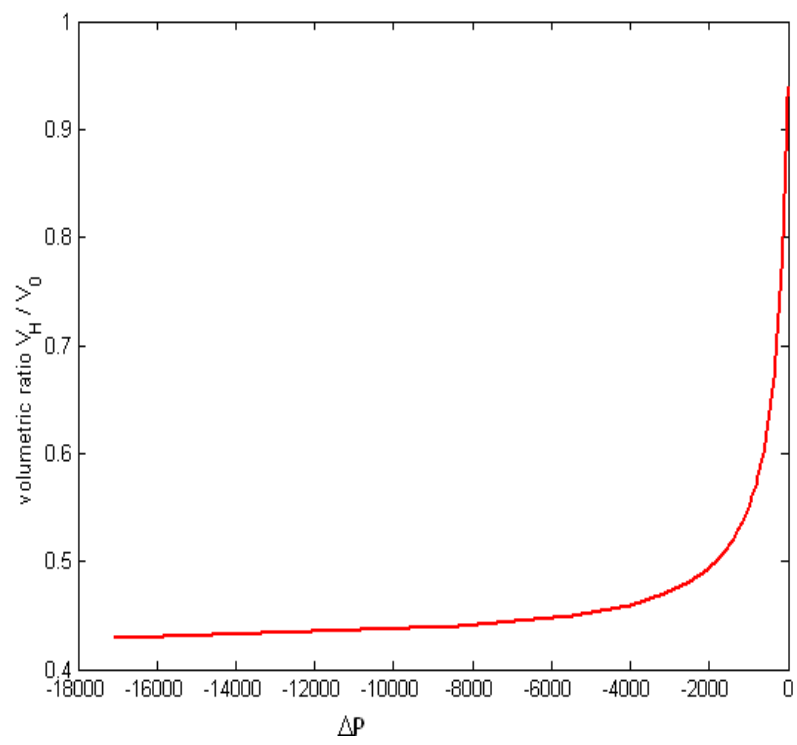

Figure 2. The change in osmotic pressure versus volumetric ratio of red blood cell with sickle cell disease from equation (24)

\section{Result and Discussions}

Figure (1) and (2) presents respectively, the viscoelastic and osmotic properties of the RBC anomalous with sickle cell disease.

Figure (1) Describes the graph of the final simplified viscoelastic constitutive function that derived from the proposed governing equation which characterize the time- dependant response of the erythrocyte membrane undergoing finite deformation elastically in uniaxial extension under normal and sickle cell disease conditions.

From our analytical solution, we test the movement of the curves by elevated the numerical values of both parameters (i.e. membrane shear elastic modulus $\mu$ and membrane viscosity $\eta_{m}$ ) and decreased strain rate $D \lambda_{1}$, assuming that $\mathrm{HbS}$ polymerization occurred in sickle cell erythrocytes, thus, the numerical simulation describes the curves of sickle erythrocytes differ from and above the normal one as shown in the figure. These curves clearly presented that, as we move from curve $\mathrm{A}$ to $\mathrm{C}$ the behavior of the cell become more rigid and poorly deformable. This analysis is consistent as reported on published literature, that both $\mu$ and $\eta_{m}$ similarly elevated above normal for ISC membrane elasticity due to an irreversible alteration in membrane structure[28]. The movement from curve (A) to (C), illustrates that, the behavior of the cell represented by curve (B) is less abnormal than that represented by curve $(C)$.

For ISC alteration in membrane structure, $\mu$ increased by $130 \%$ similarly $\eta_{m}$ also increased approximately with $150 \%$ as experimentally investigated by Nash et al,[28]. Based on such information, we calculate the value of the 
parameters as $\mu=0.023 \mathrm{dyn} / \mathrm{cm}, \eta_{m}=0.0025 \mathrm{dyn} \mathrm{s} / \mathrm{cm}$, and let $D \lambda_{1}=0.01 \mathrm{~S}^{-1}$, which gave curve (C).Therefore, (C) represents an irreversible alteration in sickle erythrocyte membrane structure. Consequently, the sickle erythrocytes membrane going to damage, hemolysis, and impaired microcirculation. In general, the study of the viscoelastic property of sickle $\mathrm{RBC}$ is used to ascertain the relative contributions of RBC membrane and the internal HbS solution to viscous and elastic components of total deformability.

Figure (2) depicts that the osmotic effect of the sickle erythrocyte by employing the osmotic pressure versus volume relation of the cell. As the curve indicates, on the graph, the gradient of osmotic pressure increases in magnitude negatively associated with decreasing cell volume, along non-linear path and gradually remains constant. This reduction behavior of the change in osmotic pressure of the cell demonstrates that, the sickle erythrocyte behaves: shrink, compression, dehydrate, and increasing polymerization of $\mathrm{HbS}$, in general it resembles that the cell undergoes in irreversible structural change and consequently leads to decreasing cell deformability.

Based on such information, totally the figure describes, the affected erythrocyte with sickle hemoglobin disease gradually tends to hemolysis, vaso-occlussion and also membrane damage.

Since the minimum value of the volumetric ratio (i.e. $V_{H} /$ $V_{0}=0.42$ ), the curve on the figure after some point, it shows almost parallel to the horizontal axis (axis of osmotic pressure). This indicates that, the cell becomes highly shrunk, compressed and dense, resulting, the cell shows more rigid. Thus, in conclusion, we can also quantify the rheological/ mechanical characterization of sickle erythrocytes by analysing its osmotical behavior.

\section{Conclusions}

In this study, we employ the generalized Voigt-model of nonlinear viscoelastic constitutive function and Van't Hoff law to characterize the deformability or rheological properties of RBCs patient with sickle cell disease. We used a Neo-Hookean strain energy function based on membrane theory for elastic part and also applied theory of viscous fluid for dissipative part of the viscoelastic constitutive relation. In this relation, the effect of mechanical properties of sickle $\mathrm{RBC}$ can be determined by varying the estimated values of the parameters above normal depend on the given data from published literature[28]. Additionally, the Van't Hoff law, used to identify the osmotic behavior by osmotic pressure -volume relations, and obtained decreasing change in osmotic pressure along with decreasing of the red cell volume in sickle cell disease. The result of both proposed mathematical formulations contribute to show the abnormal rheological behavior of the cell patient with sickle hemoglobin disease. The knowledge of this abnormal behavior has the potential to offer new diagnostic tools and treatments of the Sickle cell disease, especially for clinicians. We can also examine the abnormality of blood cell's with such disease by considering flow theory of red cell particles in our future work.

\section{REFERENCES}

[1] Brandao MM, Fontes A, Barjas-Castro ML, Barbosa LC, Costa FF, Cesar CL, Saad STO ,Optical tweezers for measuring red blood cell elasticity: application to the study of drug response in sickle cell disease. Eur J Hae matol 2003: 70: 207-211.

[2] S.Suresh, Mechanical response of human red blood cells in health and disease: Some Structure-property-function relationships. (Reviews) J. Mater. Res., Vol. 21, No. 8, 2006, 1871-1877.

[3] Ferrone, F.A., 2004. Polymerization and sickle cell dis ease: a molecular view. Microcirculation 11 (2), 115-128.

[4] JS. Gibson, and JC. Ellory, Membrane Transport in Sickle Cell Disease. Blood Cells, Molecules, and Diseases (2002) 28(3), 303-314.

[5] JL.Maciaszek, G.Lykotrafitis .Sickle cell trait human erythrocytes are significantly stiffer than normal. Journal of Biomechanics, 44 (2011), 657-661.

[6] Turner, M.S., Wang, J.C., et al., 2002. Fluctuations in selfassembled sickle hemo- globin fibers. Langmuir 18 (19), 7182-7187.

[7] C. Dong, RS. Chadwick and AN. Schechter. Influence of sickle hemoglobin polymerization and membrane proper ties on deformability of sickle erythrocytes in the micro circulation. Biophys. J. Vol. 63, 1992, 774-783.

[8] RP Hebbel, Beyond hemoglobin polymerization: the red blood cell membrane and sickle disease pathophysiology. Blood, Vol. 77, No. 2, 1991, pp .214-237.

[9] VL. Lew and RM. Bookchin. Osmotic Effects of Protein Polymerization: Analysis of Volume Changes in Sickle Cell Anemia Red Cells following Deoxy-Hemoglobin S Polymerization. J. Membrane Biol. 122, 55-67 (1991).

[10] Perutz. M.F., Mitchison, J.M. 1950.State of haemoglo bin in sickle cell anaemia. Nature, 166:677-679.

[11] Chien, S., R. G. King, A. A. Kaperonis, and S. Usami. Viscoelastic properties of sickle cells and hemoglobin. Blood Cells (NY). 8:53-64,1982.

[12] Drasler, W. J., M. S. Clark, and H. K. Keller. Viscoelas tic properties of the oxygenated sickle erythrocyte mem brane. Biorheology.1989, 26:935-949.

[13] JC .Wang, MS.Turner, G. Agarwal, S.Kwong, R.Josephs, FA. Ferrone and RW. Briehl. Micromechanics of Iso lated Sickle Cell Hemoglobin Fibers: Bending Moduli and Persistence Lengths. J. Mol. Biol. (2002) 315, 601-612.

[14] EA.Evans and RM.Hochmuth. Membrane viscoelastic ity, Biophysical Journal Vol.16, 1976.

[15] MC. Martorana, G. Mojoli, P Cianciulli, A.Tarzia, E Mannella, and P. Caprari. Sickle cell anemia: haemor heological 
aspects. Ann Ist Super Sanità 2007 | Vol. 43, No. 2: 164- 170.

[16] R.Messmann, S.Gannon, S.Sarnaik, and, R.M. Johnson. Mechanical Properties of Sickle Cell Membranes. Blood, Vol.75, No.8, 1990:pp 1711-1717.

[17] Y.Tan, D. Sun, W. Huang .Mechanical Modeling of Red Blood Cells during Optical Stretching. J.Biomechanical Engineering, 2010.132 , pp. 04450(1-5).

[18] GA.Barabino, MO.Platt, and DK.Kaul. Sickle cell Bio mechanics. Annu. Rev. Biomed. Eng. 2010. 12:345-67.

[19] E.Pucci and G.Saccomandi. On special class of Non li near Viscolelastic solids. Mathematics and Mechanics of solids, 15:803-811, 2010.

[20] M.A.J. Chaplain, and B.D.Spleeman. Modeling the growth of solid tumours and incorporating a method for their clas sification using nonlinear elasticity theory, J.Math.Biol.31; 1993, 431-473.

[21] P.Bagchi, P.Johnson, and AS.Popel. Computational Fluid Dynamic Simulation of Aggregation of Deformable Cells in a Shear Flow. J.Biomededical engineering, Vol.127, 2005; 1070-1080.

[22] M.Diez-Silva, M.Dao, J.Han, C.T. Lim, S.Suresh. Shape and Biomechanical Characteristics of Human Red Blood Cells in Health and disease.MRS Bull, 2010, 35(5), pp.382-388.

[23] Barthes-Biesel, D., Diaz, A., and Dhenin, E., 2002. Effect of Constitutive Laws for Two-dimensional Membranes on Flow-induced Capsule Deformation. J. Fluid Mech, 460, Pp, 211-222.

[24] R.Skalak, A.Tozeren, RP.Zarda, and S.Chien. Strain energy function of Red blood Cell Membranes. Biophysi cal Journal, Vol.13,1973.
[25] Dao, C.T.Lim, S. Suresh .Mechanics of the human red blood cell deformed by optical Tweezers.Journal of the mechanics and physics of solids , 2003,51, pp. 2259-2280.

[26] Mills JP, Qie L, Dao M, Lim CT, and Suresh S (2004). Nonlinear elastic and viscoelastic deformation of the human red blood cell with optical tweezers. MCB 1 (3), pp.169-180.

[27] GB Nash,CS Johnson and HJ Meiselman. in sickle cell disease Influence of oxygen tension on the viscoelastic behavior of red blood cells. Blood, 1986, 67: 110-118.

[28] GB. Nash, CS. Johnson, and HJ. Meiselman .Mechanical Properties of Oxygenated Red Blood Cells in Sickle Cell (HbSS) Disease. Blood. Vol. 63, No. 1 (January), 1984: pp. 73-82.

[29] DR. Masys, PA. Bromberg and SP. Balcerzak. Red Cells Shrink During Sickling. Blood, 1974, 44: 885-889.

[30] P.B.Canham and D.R. Parkinson, The area and volume of single human erythrocytes during gradual osmotic swelling to hemolysis, Canad.J.Phsio.Pharmaco, vol. 48, 1970, pp. 369-376.

[31] Dick.DAT. Osmotic properties of living cells. Int. Rev. Cytol.8, 1959, pp.387-448.

[32] MH.Steinberg. Pathophysiology of sickle cell disease. Baillire Clinical Haematology Vol.11, No.1, 1998: 163-184.

[33] AR. Hargens, LJ.Bowie, D.Lent, S.Carreathers, RM. Peers, HT.Hammel, and PF.Scholander. Sickle-cell hemoglobin: Fall in osmotic pressure upon deoxygenation. Proc. Natl. Acad. Sci. USA, Vol. 77, No. 7, pp. 4310-4312, 1980. 